\title{
Evaluation of the Use of Monte Carlo Calculation Programs for Quantitative Electron Probe Analysis: 1. Accuracy of Backscattered Electron Calculations
} *J. T. Armstrong

*American University, Department of Chemistry, 4400 Massachusetts Ave., NW, Washington, DC 20016; AAES Consulting, 9112 Roundleaf Way, Gaithersburg, MD 20879

Monte Carlo calculations are becoming increasingly used in electron microprobe analysis. Once a tool of the specialist who had access to high speed computing capabilities, a tool used primarily to demonstrate the nature of electron-specimen interactions in pure metals; over the past twenty years Monte Carlo programs have become readily-available, fast-to-run, and simple-to-use for the analyst [e.g., 1]. A variety of commercial and freeware stand-alone Monte Carlo programs are available, and some companies now include Monte Carlo programs in their commercial microscopy and x-ray analysis software. Investigators are starting to propose the use of Monte Carlo algorithms to directly process microprobe analysis data. Before this should happen, however, Monte Carlo algorithms need to be tested to determine that they produce results of at least comparable accuracy to those obtained by currently used methods. Moreover, the various Monte Carlo programs that claim to be using the same model and parameters should produce the same results.

In the first of a series of papers examining the state of the art of Monte Carlo methods, we address the question whether current Monte Carlo algorithms for electron interactions in solids accurately predict the nature and degree of backscattered electron production as functions of specimen composition and electron beam energy. Existing published experimental measurements of backscattered electron coefficients have been tabulated by Joy [2]. These are compared with single scattering Monte Carlo calculations using Rutherford and Mott scattering cross sections as well as plural scattering calculations. Programs examined include public domain programs made available by R. Gauvin, D. Drouin, D. Joy, NIST, and this author. Figure 1 shows typical results using Mott and Rutherford scattering models with the WinCASINO program of Drouin et al. based on the earlier CASINO program [3]. The programs tested produced comparable results when using the same scattering model. For many elements the scatter of experimental data makes it difficult to evaluate the models. Overall, the data show that Rutherford scattering should not be used to model electron scattering below $\sim 5-10 \mathrm{keV}$. Mott and Rutherford scattering give similar results for high- $Z$ elements at beam energies above $15-20 \mathrm{keV}$. Mott scattering best fits the data for mid-to-high- $Z$ elements at voltages below $5 \mathrm{keV}$, but appears to underestimate the low energy backscattering at very low $\mathrm{Z}$ materials. No consistency was found among the experimental measurements reported for multi-element compounds. There is still a need to refine data-mining criteria for existing backscatter measurements as well as making additional absolute and relative backscatter coefficients measurements for well prepared samples.

\section{References}

[1] D.C. Joy, Monte Carlo Modeling for Electro Microscopy and Microanalysis, Oxford University Press, New York (1995).

[2] D.C. Joy, Scanning 17 (1995) 270-275.

[3] D. Drouin,WinCASINO (http://www.gel.usherb.ca/casino/); P. Hovington, P. Drouin, and R. Gauvin, Scanning 19 (1997) 1-14. 

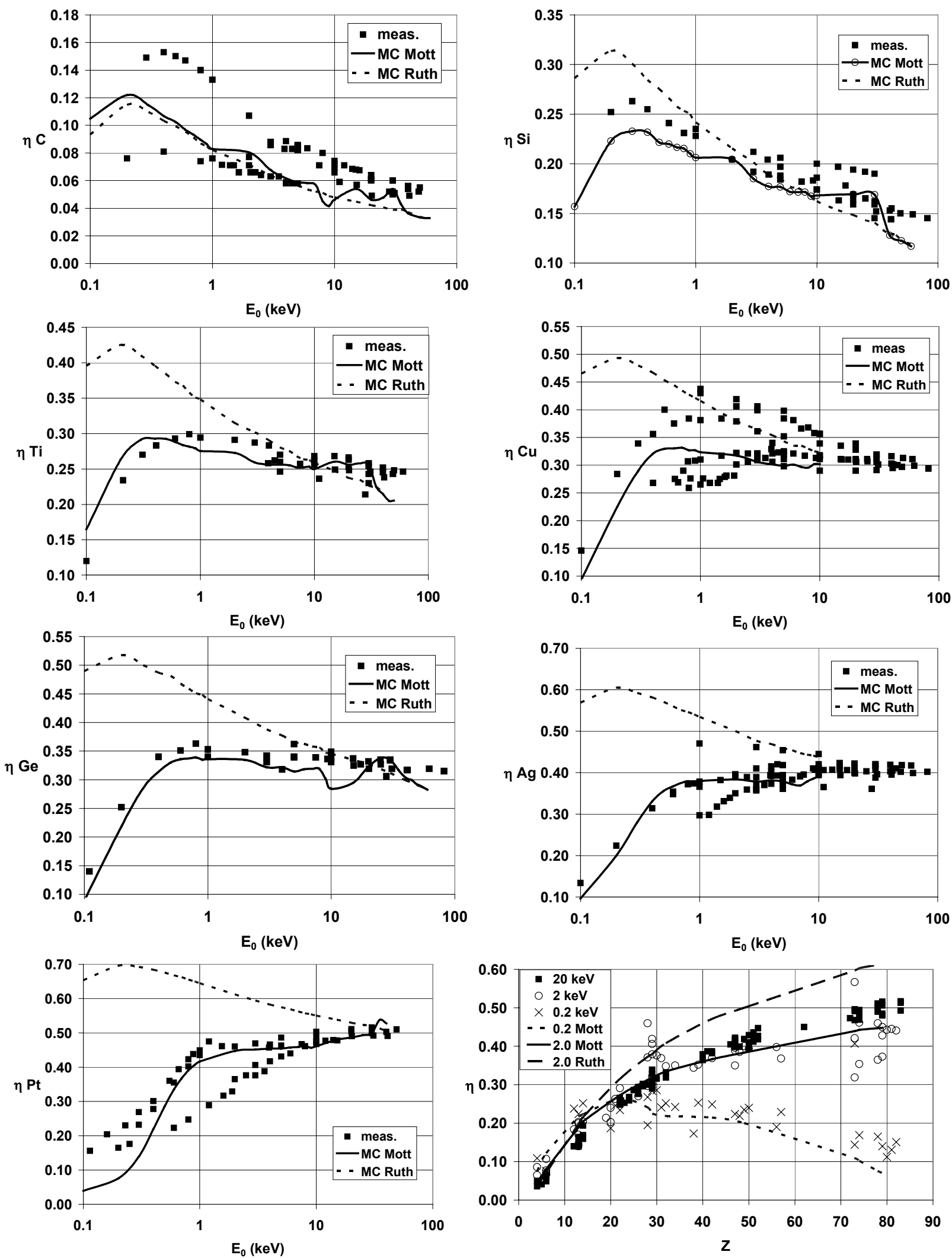

Table 1. Plots of measured and Monte Carlo calculate backscattered electron coefficients as a function of $\mathrm{E}_{\mathrm{o}}$ for $\mathrm{C}, \mathrm{Si}, \mathrm{Ti}, \mathrm{Cu}, \mathrm{Ge}, \mathrm{Ag}$, and $\mathrm{Au}$; and $\eta$ vs $\mathrm{Z}$ for $0.2,2$ and $20 \mathrm{keV}$. 